PROCEEDINGS OF THE AMERICAN MATHEMATICAL SOCIETY

Volume 124, Number 7, July 1996

\title{
TAYLOR SPECTRAL INVARIANCE FOR CRISSCROSS COMMUTING PAIRS ON BANACH SPACES
}

\author{
SHAOKUAN LI
}

(Communicated by Palle E. T. Jorgensen)

\begin{abstract}
A theorem on the commuting property of Taylor's spectrum for crisscross commuting pairs is proved in this paper.
\end{abstract}

It is well known that for two operators $A$ and $B$ on a Banach space $X$ equality $\sigma(B A) \backslash\{0\}=\sigma(A B) \backslash\{0\}$ holds; that is, $I-B A$ is invertible if and only if $I-A B$ is invertible and

$$
(I-B A)^{-1}=I+B(I-A B)^{-1} A .
$$

In the paper [3], while Crimus and Ecker study the set of matrices which can be simultaneously diagonalized by two unitary matrices, the property of crisscross commuting is introduced and it guarantees that the tuples of multiplication operators are commuting, i.e., if $A$ and $B$ are crisscross commuting pairs, then $A B$ and $B A$ are commuting tuples of operators. This may have some applications to mathematical physics.

In the paper [2] we proved that for crisscross commuting pairs $A=\left(A_{1}, \ldots, A_{n}\right)$ and $B=\left(B_{1}, \ldots, B_{n}\right)$ it holds that

$$
\mathrm{Sp}(A B) \cup\{(0, \ldots, 0)\}=\operatorname{Sp}(B A) \cup\{(0, \ldots, 0)\},
$$

where $A B=\left(A_{1} B_{1}, \ldots, A_{n} B_{n}\right)$ and $\operatorname{Sp}(\cdot)$ denotes Taylor's spectrum. In this paper we will discuss the problem for Taylor's essential spectrum.

First, we introduce some notation.

Let $\bigwedge_{n}(e)$ be the exterior algebra generated by $e_{1}, \ldots, e_{n}$ and $O_{n}=\left\{I=\left\{i_{1}<\right.\right.$ $\left.\left.i_{2}<\cdots<i_{k}\right\}: I \subset\{1, \ldots, n\}\right\}$. For $I \in O_{n}$ we denote $e_{I}=e_{i 1} \cdots e_{i k}$. Note that when $I=\Phi, e_{I}=e_{\Phi}=1$. In this case, $\bigwedge_{n}(e)$ becomes a Hilbert space with orthonormal basis $\left\{e_{I}: I \in O_{n}\right\}$. If we denote $\bigwedge_{p}(e)=\operatorname{Span}\left(e_{I}: I \in O_{n},|I|=p\right)$, then $\bigwedge_{n}(e)=\bigoplus_{p=0}^{n} \Lambda^{p}(e)$. In the Hilbert space $\bigwedge_{n}(e)$ we define operators $E_{i}$, $i=1,2, \ldots, n$, by $E_{i} x=e_{i} x$. It is obvious that

$$
E_{i} E_{j}+E_{j} E_{i}=0, \quad E_{i}^{*} E_{j}+E_{j} E_{i}^{*}=\delta_{i} I .
$$

Received by the editors May 2, 1994 and, in revised form, September 30, 1994, November 29, 1994, and January 7, 1995.

1991 Mathematics Subject Classification. Primary 47A10.

Key words and phrases. Tuple of operators, Taylor's spectrum, Fredholm tuple.

(C)1996 American Mathematical Society 
Let $T=\left(T_{1}, \ldots, T_{1}\right)$ be a commuting tuple of the operators on Banach space $X$. Corresponding to $T$, there is a complex sequence

$$
D_{T}: 0 \frac{D_{-1}}{} X \otimes \bigwedge^{0}(e) \frac{D_{0}}{2} \cdots \frac{D_{n-1}}{} X \otimes \bigwedge^{n}(e) \frac{D_{n}}{-} 0
$$

where $D_{T}=\sum_{i=1}^{n} T_{i} \otimes E_{i}$. If $\operatorname{ker}\left(D_{k}\right)=R\left(D_{k-1}\right)$ for $k=0,1, \ldots, n$, then $T$ is nonsingular. If $R\left(D_{k-1}\right)$ is closed and $\operatorname{dim} \operatorname{ker}\left(D_{k}\right) / R\left(D_{k-1}\right)<\infty$ for $k=0,1, \ldots, n$, then $T$ is Fredholm with its index defined by $\operatorname{ind}(T)=\sum_{K=0}^{n}(-1)^{k} \operatorname{dim} H^{k}(T)$, where $H^{k}=\operatorname{Ker}\left(D_{k}\right) / R\left(D_{k-1}\right)$. Of course, we can consider $D_{T}$ as an operator on $X \otimes \bigwedge_{n}(e)$. It is obvious that $T$ is Fredholm if and only if $R\left(D_{T}\right)$ is closed and $\operatorname{dim} \operatorname{ker}\left(D_{T}\right) / R\left(D_{T}\right)$ is finite.

The following is the main result of this note:

Theorem 1. Let $A=\left(A_{1}, \ldots, A_{n}\right)$ and $B=\left(B_{1}, \ldots, B_{n}\right)$ be crisscross commuting pairs on Banach space $X$, i.e., they satisfy the conditions:

$$
A_{i} B_{j} A_{k}=A_{k} B_{j} A_{i}, \quad B_{i} A_{j} B_{k}=B_{k} A_{j} B_{i} .
$$

Let $T=\left(\varepsilon_{1}-A_{1} B_{1}, \ldots, \varepsilon_{n}-A_{n} B_{n}\right)$ and $S=\left(\varepsilon_{1}-B_{1} A_{1}, \ldots, \varepsilon_{n}-B_{n} A_{n}\right)$, where there exists at least one $k$ such that $\varepsilon_{k} \neq 0$. Then $T$ is Fredholm if and only if $S$ is Fredholm and in this case

$$
\operatorname{ind}(T)=\operatorname{ind}(S), \quad \operatorname{dim} H^{k}(T)=\operatorname{dim} H^{k}(S), \quad k=0,1, \ldots, n .
$$

Proof. Without loss of generality, we may suppose $\varepsilon_{1}=1$ and denote

$$
D_{1}=\sum_{K=1}^{n}\left(\varepsilon_{k}-A_{k} B_{k}\right) \otimes E_{k}, \quad D_{2}=\sum_{K=1}^{n}\left(\varepsilon_{k}-B_{k} A_{k}\right) \otimes E_{k} .
$$

We can regard $A_{i}$ and $B_{i}$ as operators on $X \otimes \bigwedge_{n}(e)$ by $A_{i}\left(x \otimes e_{I}\right)=A_{i} x \otimes e_{I}$ and $B_{i}\left(x \otimes e_{I}\right)=B_{i} x \otimes e_{I}$. It is easy to know $A_{i} D_{2}=D_{1} A_{i}$ and $B_{i} D_{1}=D_{2} B_{i}$ from the conditions.

If $T$ is Fredholm, then there exist a finite-dimensional subspace $M$ such that $\operatorname{ker}\left(D_{1}\right)=R\left(D_{1}\right)+M$, i.e., $M$ is equal to $H(T)$. Suppose $x \in \operatorname{ker}\left(D_{2}\right)$. From $A_{1} D_{2}=D_{1} A_{1}$ we know that $D_{1} A_{1} x=A_{1} D_{2} x=0$, i.e., $A_{1} x \in \operatorname{ker}\left(D_{1}\right)$. Therefore there exist a vector $y \in X \otimes \bigwedge_{n}(e)$ and $u \in M$ such that

$$
A_{1} x=D_{1} y+u \text {. }
$$

Let $v=B_{1} y+E_{1}^{*} x$; then we have

$$
\begin{aligned}
D_{2} v & =D_{2} B_{1} y+D_{2} E_{1}^{*} x \\
& =B_{1} D_{1} y+\sum_{k=1}^{n} S_{k} \otimes E_{1}^{*} x \\
& =B_{1} A_{1} x-B_{1} u-\sum_{k=1}^{n} S_{k} \otimes E_{1}^{*} E_{k} x+S_{1} x \\
& =B_{1} A_{1} x-B_{1} u-E_{1}^{*} D_{2} x+\left(I-B_{1} A_{1}\right) x \\
& =x-B_{1} u .
\end{aligned}
$$

Thus we get

$$
x=D_{2} v+B_{1} u .
$$

Therefore $R(D)$ has finite codimension in $\operatorname{ker}\left(D_{2}\right)$ and $\operatorname{dim} \operatorname{ker}\left(D_{2}\right) / R\left(D_{2}\right) \leqslant$ $\operatorname{dim} B_{1} M \leqslant \operatorname{dim} H(T)$. Thus $S$ is Fredholm and $\operatorname{dim} H(S) \leqslant \operatorname{dim} H(T)$. From 
symmetry, we know that $\operatorname{dim} H(S)=\operatorname{dim} H(T)$, and $B_{1}$ is bijective from $H(T)$ onto $H(S)$. It is obvious that $B_{1}$ is also one-to-one and maps $H^{k}(T)$ onto $H^{k}(S)$ for $k=0,1, \ldots, n$. Thus the relations (5) are proved.

Corollary 2. Under the conditions of Theorem 1 we have

$$
\operatorname{Sp}_{e}(A B) \cup\{(0, \ldots, 0)\}=\operatorname{Sp}_{e}(B A) \cup\{(0, \ldots, 0)\}
$$

where $\operatorname{Sp}_{e}(A)$ is Taylor's essential spectrum of $A$.

In particular we have

Corollary 3. For operators $A$ and $B$ on Banach space, $(I-A B)$ is Fredholm if and only if $(I-B A)$ is Fredholm and in this case

$$
\begin{gathered}
\operatorname{ind}(I-A B)=\operatorname{ind}(I-B A), \quad \operatorname{dim} \operatorname{ker}(I-A B)=\operatorname{dim} \operatorname{ker}(I-B A) . \\
\text { REFERENCES }
\end{gathered}
$$

\section{REFERENCES}

1. R. E. Curto and L. Fialkow, The spectral pictures of $\left(L_{A}, R_{B}\right)$, J. Funct. Anal. 71 (1989), 371-392. MR 88c: 47006

2. Shaokuan Li, On the commuting properties of Taylor's spectrum, Chinese Sci. Bull. 37 (1992), 1849-1852.

3. W. Crimus and C. Ecker, On the simultaneous diagonalizability of matrices, J. Phys. A 9 (1986), 3917-3919.

Department of Basic Sciences, China Textile University, 200051, Shanghai, People's Republic of China 